April $\quad 14, \quad 2016 \quad 0: 24 \quad$ WSPC/INSTRUCTION $\quad$ FILE
MG14CM2

International Journal of Modern Physics D

(C) World Scientific Publishing Company

\title{
RECENT RESULTS AND PERSPECTIVES ON COSMOLOGY AND FUNDAMENTAL PHYSICS FROM MICROWAVE SURVEYS*
}

\author{
CARLO BURIGANA ${ }^{1,2,3, a}$, ELIA STEFANO BATTISTELLI ${ }^{4, b}$, MICOL BENETTI $^{5, c}$, \\ GIOVANNI CABASS ${ }^{4, d}$, PAOLO DE BERNARDIS ${ }^{4, e}$, \\ SPERELLO DI SEREGO ALIGHIERI ${ }^{6, f}$, ELEONORA DI VALENTINO $^{7, g}$, \\ MARTINA GERBINO ${ }^{8,9,4, h}$, ELENA GIUSARMA ${ }^{4, i}$, ALESSANDRO GRUPPUSO $^{1,3, j}$, \\ MICHELE LIGUORI ${ }^{10,11, k}$, SILVIA MASI ${ }^{4, l}$, HANS ULRIK NORGAARD-NIELSEN ${ }^{12, m}$, \\ PIERO ROSATI ${ }^{2, n}$, LAURA SALVATI ${ }^{4, o}$, \\ TIZIANA TROMBETTI ${ }^{1,2, p}$, PATRICIO VIELVA ${ }^{13, q}$ \\ ${ }^{1}$ INAF-IASF Bologna, Via Piero Gobetti 101, I-40129 Bologna, Italy ${ }^{\dagger}$ \\ ${ }^{2}$ Dipartimento di Fisica e Scienze della Terra, Università degli Studi di Ferrara, \\ Via Giuseppe Saragat 1, I-44122 Ferrara, Italy \\ ${ }^{3}$ INFN, Sezione di Bologna, Via Irnerio 46, I-40126, Bologna, Italy \\ ${ }^{4}$ Dipartimento di Fisica e INFN, Università di Roma "La Sapienza", \\ P.le Aldo Moro 2, 00185, Rome, Italy \\ ${ }^{5}$ Observatório Nacional, 20921-400, Rio de Janeiro, RJ, Brazil \\ ${ }^{6}$ INAF-Osservatorio Astrofisico di Arcetri, Largo Enrico Fermi 5, I-50125 Firenze, Italy \\ ${ }^{7}$ Institut d'Astrophysique de Paris \\ (UMR7095: CNRS \&S UPMC - Sorbonne Universities), F-75014, Paris, France \\ ${ }^{8}$ The Oskar Klein Centre for Cosmoparticle Physics, Department of Physics, \\ Stockholm University, AlbaNova, SE-106 91 Stockholm, Sweden \\ ${ }^{9}$ Nordita (Nordic Institute for Theoretical Physics), Roslagstullsbacken 23, SE-106 91 \\ Stockholm, Sweden \\ ${ }^{10}$ Dipartimento di Fisica e Astronomia G. Galilei, Università degli Studi di Padova, \\ Via Marzolo 8, 35131 Padova, Italy \\ ${ }^{11}$ INFN, Sezione di Padova, via Marzolo 8, I-35131 Padova, Italy \\ ${ }^{12}$ DTU Space, Elektrovej, DK - 2800 Kgs. Lyngby, Denmark \\ ${ }^{13}$ Instituto de Física de Cantabria (CSIC-UC), Santander, 39005, Spain \\ ${ }^{a}$ burigana@iasfbo.inaf.it - ${ }^{b}$ elia.battistelli@roma1.infn.it $-{ }^{c}$ micolbenetti@on.br \\ ${ }^{d}$ giovanni.cabass@gmail.com - ${ }^{e}$ paolo.debernardis@roma1.infn.it - ${ }^{f}$ sperello@arcetri.astro.it \\ ${ }^{g}$ valentin@iap.fr $-{ }^{h}$ martina.gerbino@uniroma1.it $-{ }^{i}$ elena.giusarma@roma1.infn.it \\ ${ }^{j}$ gruppuso@iasfbo.inaf.it $-{ }^{k}$ michele.liguori@pd.infn.it $-{ }^{l}$ silvia.masi@roma1.infn.it \\ ${ }^{m}$ hunn@space.dtu.dk - ${ }^{n}$ rosati@fe.infn.it - ${ }^{o}$ laura.salvati@roma1.infn.it \\ ${ }^{p}$ trombetti@iasfbo.inaf.it_- ${ }^{q}$ vielva@ifca.unican.es
}

Received 1 March 2016

Accepted 2 March 2016

Recent cosmic microwave background data in temperature and polarization have reached high precision in estimating all the parameters that describe the current so-called stan-

\footnotetext{
*Based on presentations at the Fourteenth Marcel Grossmann Meeting on General Relativity, Rome, July 2015.

$\dagger$ Istituto Nazionale di Astrofisica - Istituto di Astrofisica Spaziale e Fisica Cosmica di Bologna, Via Piero Gobetti 101, I-40129 Bologna, Italy
} 
dard cosmological model. Recent results about the integrated Sachs-Wolfe effect from cosmic microwave background anisotropies, galaxy surveys, and their cross-correlations are presented. Looking at fine signatures in the cosmic microwave background, such as the lack of power at low multipoles, the primordial power spectrum and the bounds on non-Gaussianities, complemented by galaxy surveys, we discuss inflationary physics and the generation of primordial perturbations in the early Universe. Three important topics in particle physics, the bounds on neutrinos masses and parameters, on thermal axion mass and on the neutron lifetime derived from cosmological data are reviewed, with attention to the comparison with laboratory experiment results. Recent results from cosmic polarization rotation analyses aimed at testing the Einstein equivalence principle are presented. Finally, we discuss the perspectives of next radio facilities for the improvement of the analysis of future cosmic microwave background spectral distortion experiments.

Keywords: Cosmology; Background radiations; Radio, microwave; Origin and formation of the Universe; Particle-theory and field-theory models of the early Universe; Observational cosmology; Large scale structure of the Universe; Dark matter; Dark energy; Elementary particle processes.

PACS numbers: 98.80.-k; 98.70.Vc; 95.85.Bh; 98.80.Bp; 98.80.Cq; 98.80.Es; 98.65.Dx; 95.35.+d; 95.36.+x; 95.30.Cq.

\section{Introduction}

Latest measurements of cosmic microwave background (CMB) anisotropies in temperature and polarization from Planck satellite complemented at smaller scales by recent ground-based experiments (see e.g. Refs. [2, 3, 4, 5]) and combined with other cosmological information coming from e.g. type-Ia supernovae, galaxy and galaxy cluster surveys, have reached high precision in estimating all the parameters that describe the current so-called standard cosmological model. Far from representing a fully, physically exhaustive interpretation of the Universe properties, the cosmological constant plus cold dark matter $(\Lambda \mathrm{CDM})$ model phenomenologically describes reasonably well existing data with a simple set of six parameters (see e.g. the lectures by M. Bersanelli and J.-L. Puget on Planck result: ${ }^{\mathrm{b}}$ in this Meeting). The integrated Sachs-Wolfe effect, discussed here in Sect. 2 represents a remarkable example of the success of current cosmology, since a such intrinsically weak predicted signal is clearly recognized in two classical cosmological probes, like $\mathrm{CMB}$ anisotropies and galaxy surveys, and in their cross-correlations. Looking at fine signatures in the CMB it is possible to derive more hints on early Universe and inflationary physics as well as to carry out a sort of laboratory tests to constrain particle and fundamental physics. In Sect. 3 the "lack of power" in the large

\footnotetext{
${ }^{a}$ Planck is a project of the European Space Agency - ESA - with instruments provided by two scientific Consortia funded by ESA member states (in particular the lead countries: France and Italy) with contributions from NASA (USA), and telescope reflectors provided in a collaboration between ESA and a scientific Consortium led and funded by Denmark.

${ }^{\mathrm{b}}$ This paper is based largely on the products available at the ESA Planck Legacy Archive and publicly available publications by ESA and the Planck Collaboration, for what concerns the related aspects. Any material presented here that is not already described in Planck Collaboration papers represents the views of the authors and not necessarily those of the Planck Collaboration.
} 
scale pattern (i.e. low multipole region) of CMB anisotropy angular power spectrum (APS) is investigated to link the inflationary phase to the string theory while CMB data and galaxy surveys are jointly analyzed in Sect. 4 to constrain inflationary models predicting localized 'features' in the primordial power spectrum (PPS). Going beyond power spectrum (PS) analyses, the study of primordial non-Gaussianity (PNG), discussed in Sect. 5, allows to test mechanisms for the generation of primordial perturbations in the early Universe. Sects. 6 and 7 discuss two important topics in dark matter studies, respectively the bounds on neutrinos masses and parameters and on thermal axion mass from cosmological data while Sect. 8 summarizes the state of the art on the neutron lifetime, $\tau_{n}$, a fundamental quantity in nuclear physics. Attention is given to the comparison with laboratory experiment results. Sect. 9 is devoted to the test of the Einstein equivalence principle (EEP), at the basis of general relativity (GR), through the analysis of the cosmic polarization rotation (CPR) and to the comparison of results from astronomical and CMB based analyses. Finally, Sect. 10 discusses the main cosmological and fundamental physics information contained in the CMB spectral distortions in the light of the contribution expected from the Square Kilometre Array (SKA).

\section{Integrated Sachs-Wolfe effect}

The late integrated Sachs-Wolfe (ISW) effect $\frac{\sqrt{6}}{6}$ is a secondary anisotropy in the cosmic microwave background (CMB), which is caused by the interaction of CMB photons with the time-dependent gravitational potential of the evolving cosmic large-scale structure (LSS). The ISW effect can be generated under several scenarios affecting the late evolution of the structures: a cosmological constant, dark energy (DE) ${ }^{9}$ modified gravity, 10 or spatial curvature! 11

The early ISW is generated after recombination (since the energy density of relativistic matter is still considerable at that time): it adds in phase with the Sachs-Wolfe primary anisotropy, increasing the height of the first acoustic peaks. Besides, the effect on the APS, $C_{\ell}$ (being $\ell$ the multipole of the spherical harmonic expansion), is suppressed by the factor $\rho_{\mathrm{rad}}^{2}\left(\eta_{\mathrm{rec}}\right) / \rho_{\mathrm{m}}^{2}\left(\eta_{\mathrm{rec}}\right)$ : increasing the radiation energy density with respect to that of matter near recombination will give a larger early ISW effect. The late ISW effect is active at more recent times: focusing on scales corresponding to galaxy clusters, the CMB photons get redshifted by the time-dependent gravitational potentials. The potentials causing the late ISW also give rise to the weak lensing distortions: the interplay between these two effects results in a non-Gaussian correlation between small and large angular scales, which is encoded in the lensing-induced bispectrum.

The optimal detection ${ }^{9}$ of the ISW effect is made by the cross-correlation of the CMB temperature anisotropies with tracers of the gravitational potential, like, for instance, galaxy catalogues. The first detection ${ }^{12}$ was made using Wilkinson Microwave Anisotropy Probe (WMAP) data and radio and X-ray galaxy catalogues. The ISW signal is very weak (an ideal LSS tracer could provide a detection of up to 
$\approx 8 \sigma$ ), and, therefore, its capability to constrain cosmological parameters, is very limited. Nevertheless, using the ISW signal alone it is possible to constrain some cosmological parameters, by fixing the remaining ones to their standard value (see e.g. the estimation of the DE density parameter ${ }^{13} \Omega_{\Lambda} \approx 0.67$, with an error of about $20 \%$; the compatibility of the DE equation of state parameter with the expected value for a $\Lambda \mathrm{CDM}$ scenario, $\sqrt{14}$ or the setting of upper limits on spatial flatness of a few per cent ${ }^{15}$ ).

We will focus here on the main results of the ISW effect derived by Planck (see Refs. [16] and [13] for a complete description).

\subsection{The ISW probed through the CMB-LSS cross-correlation}

The four CMB maps ${ }^{17}$ produced by Planck (COMMANDER, NILC, SEVEM, SMICA) have been cross-correlated with several tracers of the LSS. In the first release, the NRAO VLA Sky Survey (NVSS) radio-galaxy catalogue, the photometric luminous galaxy (SDSS-CMASS/LOWZ), and the photometrically-selected galaxies (SDSS-MphG) from the Sloan Digital Sky Survey (SDSS) were considered. Two additional catalogues from the Wide-Field Infrared Survey Explorer (WISE) were added to the analysis of the second release: one based on star-forming galaxies (WISE-GAL), and another one based on active galactic nuclei (WISE-AGN). Considering the full cross-correlations of the CMB with all the LSS tracers, the latest results provided a total ISW detection of around $3 \sigma$, as expected for the standard $\Lambda$ CDM model. The NVSS catalogue already provides by itself a similar detection level.

The most novel result provided by Planck was its capability to provide a detection of the ISW without relying on external tracers of the LSS, thanks to its reliable estimation of the gravitational potential through the lensing suffered by the CMB photons $\frac{18}{18}$ The cross-correlation of this map with the CMB one, or, equivalently, the specific shape of the ISW-lensing bispectrum, reported a detection of the ISW at around $\approx 3 \sigma$. When all the LSS tracers are combined, the total ISW detection is $\approx 4 \sigma$, also in good agreement with the $\Lambda \mathrm{CDM}$ model.

Assuming the standard $\Lambda$ CDM model, the statistical ISW captured in the CMBLSS cross-correlation can be used to estimate a map of the ISW anisotropies caused by the gravitational potential traced by each of the LSS probes ${ }^{[19}$ Fig. 1 shows the ISW fluctuation maps obtained from the full cross-correlation of the Planck SEVEM CMB map with NVSS, WISE-AGN, WISE-GAL, SDSS-CMASS/LOWZ, SDSSMphG, and the Planck lensing LSS tracers.

All these results were obtained without using CMB polarization information (except for the estimation of the Planck lensing map). In principle, including polarization could increase the ISW detection 20 around a 15\%, however, the current CMB polarization data from Planck is high-pass filtered at the largest angular scales $\left(\gtrsim 5^{\circ}\right)$, which are the most important ones in this context. Including the large scale polarization is, perhaps, the most important remaining aspect within the context of the ISW study, at least, from the CMB side. On the other side, future galaxy surveys 

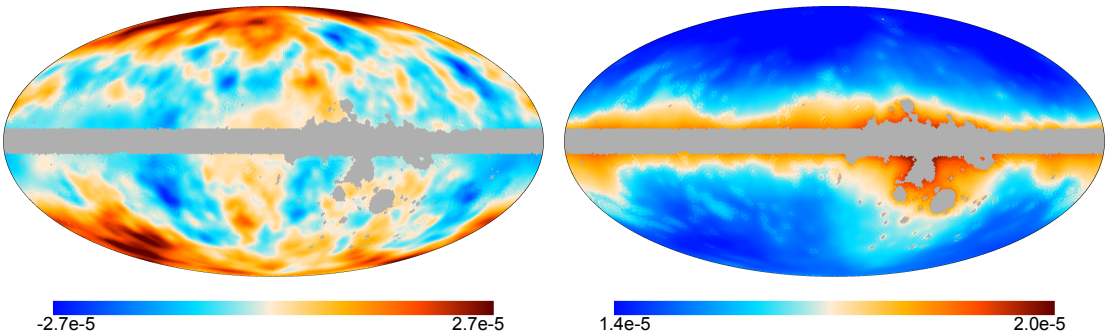

Fig. 1. Map (thermodynamic temperature in K) of the recovered ISW anisotropies (left) and its corresponding estimated error per pixel (right), from the combination of the Planck SEVEM CMB map and all the LSS tracers. The error map structure is determined by the sky coverage of the different surveys. The total signal-to-noise of the ISW map cannot exceed, obviously, a value of $\approx 4$. The signal-to-noise is higher near the Galactic poles, with values of $\approx 2$.

like Euclid, ${ }^{21} \mathrm{~J}-\mathrm{PAS} \stackrel{\sqrt{22}}{ }$ or LSST, 23 among others, will provide accurate galaxy catalogues, probing very large volumes, allowing to perform, for instance, a tomographic ISW detection.

A complementary approach consists in stacking the CMB fluctuations in the position of known structures, such as voids and clusters, as done initially on WMAP data ${ }^{24}$ using a catalogue (GR08) of super-structures from SDSS. An anomalous ISW signal, incompatible with the standard $\Lambda \mathrm{CDM}$ model was found, confirmed in the Planck analyses, showing that the intensity of the detected signal $(\approx-11 \mu \mathrm{K}$ for voids, and $\approx+8.5 \mu \mathrm{K}$ for clusters) and the scale at which that signal is maximum $\left(\approx 3.5^{\circ}\right.$ for voids, and $\approx 4.5^{\circ}$ for clusters $)$ are, indeed, unexpected.

At these scales, the current CMB Planck polarization map still retains certain signal, despite the high-pass filtering and, therefore, it can be used to test further the nature of this anomalous signal. The key point is that, if this signal is caused by the ISW effect, and, therefore, originated by a gravitational secondary CMB anisotropy, a negligible contribution of the CMB polarization is expected. In fact, no associated polarization is found in Planck data, although the diminishing of the signal caused by the high-pass filtering limits any strong conclusion. Anyway, the current polarization data are not in contradiction with assuming that the emission coming from these GR08 structures provides an anomalous ISW signal. Studying the stacked fluctuations of the Planck lensing map on the GR08 positions also supports this hypothesis. In fact, at least for the voids, a clear correlation between the lensing gravitational potential and the position of the super-structures is found.

This kind of studies could be further extended once Planck provides its next and final release, which will include polarization information at all the angular scales.

\subsection{Parametrization of early and late $I S W$ and data analysis}

The ISW amplitude can be parametrized in terms of $A_{\text {eISW }}$ and $A_{l \mathrm{ISW}}$, which rescale the contribution of the ISW to the temperature anisotropies. A Markov-chain 
Monte-Carlo (MCMC) analysis was performed with a baseline standard $\Lambda$ CDM model and flat priors on the parameters ${ }^{25}$ We also check the impact of a Gaussian prior $A_{l \mathrm{ISW}}=1.00 \pm 0.25$, consistent with the $68 \%$ confidence level (C.L.) bounds on the same parameter from the estimation of the ISW-lensing bispectrum, which has been obtained by cross-correlating the Planck CMB maps with the Planck map of the lensing potential. Various datasets were tested: the high- $\ell$ Planck temperature and temperature+polarization APS in the range $30 \leq \ell<2500$ (hereafter $T T$ and $T T, T E, E E$, respectively) in combination with the low- $\ell$ Planck temperature and polarization APS in the range $2 \leq \ell<30$ (lowP). We also tested the WMAP APS including both temperature and polarization up to $\ell=1200$.

Planck $T T+$ lowP data provide tighter constraints than WMAP on the early ISW $\left(A_{\text {eISW }}=1.064_{-0.043}^{+0.042}\right.$ vs. $A_{\text {eISW }}=1.007_{-0.058}^{+0.056}$ at $68 \%$ C.L. $)$, and present a $1 \sigma$ evidence of $A_{\text {eISW }} \neq 1$ that is stable when considering the extensions of the $\Lambda \mathrm{CDM}$ model shown in Fig. 2. Regarding the late ISW, Planck data place a constraint $A_{l \text { ISW }}<1.14$ at $95 \%$ C.L.: Planck alone does not improve significantly the constraint on $A_{l \mathrm{ISW}}$ with respect to WMAP data (which give $A_{l \mathrm{ISW}}=0.958_{-0.317}^{+0.391}$ at $68 \%$ C.L.). In fact, the late ISW affects angular scales that are dominated by cosmic variance, rather than by instrumental noise. Adding the prior on $A_{l \mathrm{ISW}}$ coming from CMB temperature anisotropies-weak lensing correlations, we find a $\sim 4 \sigma$ detection $A_{l \mathrm{ISW}}=0.85 \pm 0.21$. When we consider the recent Planck polarization data at high $\ell$, the evidences for a non-standard value of $A_{\text {eISW }}$ disappear. Using also the small scale polarization APS does not change the results obtained for $A_{l \mathrm{ISW}}$ : their effect is to tighten the upper bounds obtained considering only the $T T+$ lowP APS.

\section{CMB low multipoles anomalies}

It is usually stated that the six parameters of the $\Lambda$ CDM mode 10 26 27 are enough to describe the large scale Universe. However, some features are not well captured, and anomalies occur for instance at the largest CMB angular scales (see e.g. Ref. [28]), although they are often regarded as mere curiosities. We focus here on the lack of correlation $\sqrt[29]{37}$ and explain why it deserves attention. The low variance anomaly $\sqrt{38}$ is a closely related observation, $\frac{36}{3}$ so that the terms "lack of power" and "lack of correlation" are used as synonymous.

There is a lack of power, with respect to $\Lambda \mathrm{CDM}$, in the two-point correlation function of $\mathrm{CMB}$ temperature anisotropies for angles $\gtrsim 60^{\circ}$, as originally noted with $\mathrm{COBE}^{\mathrm{c}}$ data ${ }^{29}$ and then confirmed by the WMAP team already in their first year release $\frac{30}{30}$ In Ref. 31 this feature was associated to missing power in the quadrupole. WMAP-3yrs and WMAP-5yrs data were then used to show $32 \sqrt[33]{33}$ that a lack of correlation occurs only in $0.03 \%$ of the $\Lambda \mathrm{CDM}$ realizations. A subsequent analysis ${ }^{34}$ confirmed the anomaly using WMAP-5yrs data, and, at the same time, found, with a Bayesian approach, that the $\Lambda \mathrm{CDM}$ model cannot be excluded.

$\overline{{ }^{c} \text { http://lambda.gsfc.nasa.gov/product/cobe/ }}$ 

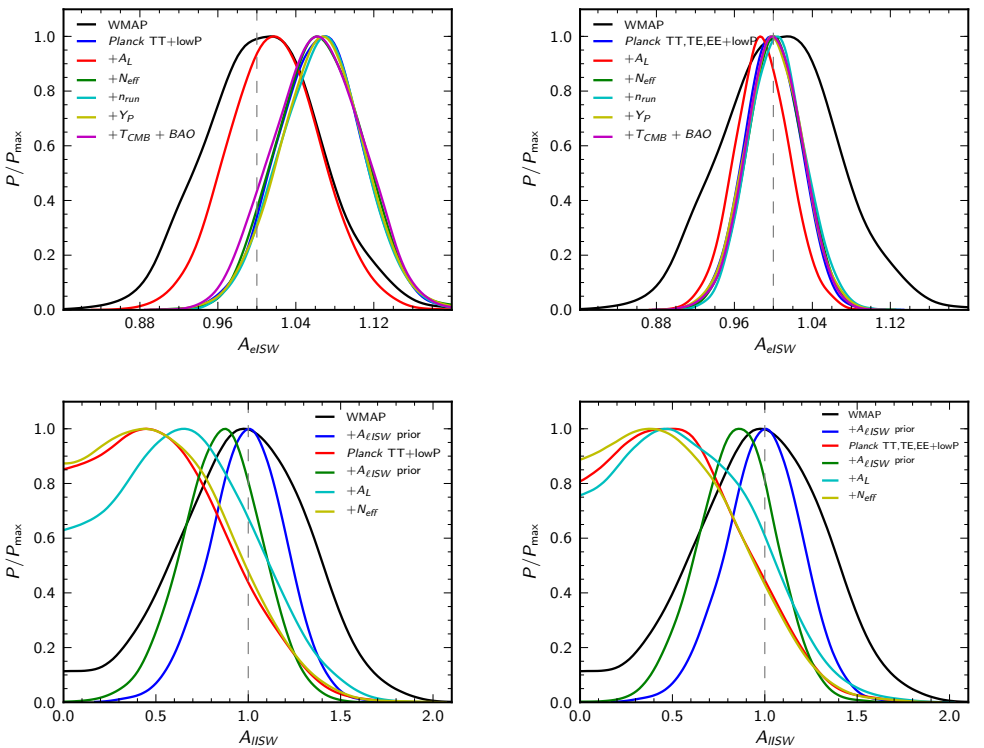

Fig. 2. One-dimensional posteriors for $A_{e I S W}$ and $A_{l I S W}$. In the left column, only Planck temperature and low multipole polarization APS (Planck $T T+$ lowP) were used. The plots in the right column use also the Planck polarization APS at high multipoles (Planck TT, TE, EE + lowP). From Ref. [25].

WMAP-7yrs data were taken into account in Ref. [35, while WMAP-9yrs data were considered in Ref. [36, where the lack of correlation was studied against the Galactic masking. Planck 2013 and WMAP-9yrs data were analyzed in Ref. 37, which confirmed for this anomaly a significance at the level of $99.97 \%$. Similar results were obtained in Ref. 39] where Planck 2015 data were taken into account. One intriguing feature of this anomaly is that it is more significant at high Galactic latitude $36[39$ Is this a simple statistical fluke or it is caused by a physical mechanism?

We now elaborate on a possible fundamental origin for this effect 40 Lack of power at large angular scales is a typical manifestation of early departures from slow-roll, which follow naturally the emergence from an initial singularity. As explained in Refs. [42, 43, when this occurs the PS approaches in the infrared the limiting behavior

$$
\mathcal{P}(k)=A \frac{k^{3}}{\left(k^{2}+\Delta^{2}\right)^{2-n_{s} / 2}},
$$

which brings along a new physical scale $\Delta$. An infrared depression of the PS presents itself naturally in string theory, in orientifold vacua with high-scale supersymmetry breaking (see e.g. references in Refs. [40, 41]). In these models a scalar field emerges at high speed from an initial singularity, to then bounce against a steep exponential potential before attaining an eventual slow-roll regime. The key ingredient is the steep exponential, whose logarithmic slope is predicted by string theory, $\stackrel{44}{a}$ and a 
number of exactly solvable systems provide explicit realizations of this peculiar dynamics. The results of a Bayesian analysis extended to all standard cosmological

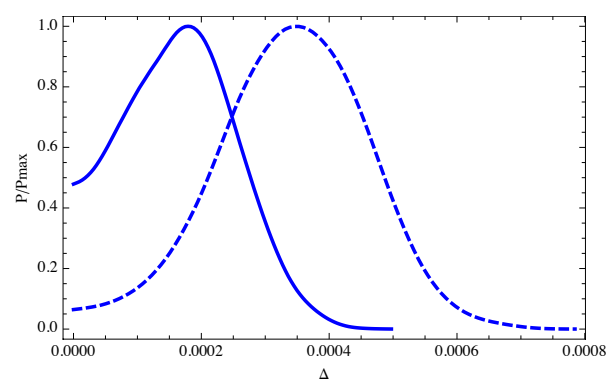

Fig. 3. Posterior probabilities of $\Delta$ (solid line for the standard mask with $f_{s k y} \simeq 90 \%$, and dashed line for an extended mask with $f_{s k y} \simeq 40 \%$ ).

parameters and based on Planck 2013 data are shown in Fig. 3, where posteriors for $\Delta$ are given for two choices of the Galactic mask (even if equivalent, for an updated and complete analysis see Ref. 41). For the latter choice, the estimated value is

$$
\Delta=(0.340 \pm 0.115) \times 10^{-3} \mathrm{Mpc}^{-1} .
$$

Interestingly, $\Delta$ in eq. $(2)$ is found to differ from 0 at $99 \%$ C.L. and its magnitude appears reasonable $\stackrel{40}{41}$ Moreover, in analogy with the lack of power anomaly, Fig. 3 shows that the significance of this result increases sizably for a larger Galactic mask.

In conclusion, the considerations in Refs. [42, 43], inspired by string theory, and in particular by the supersymmetry breaking mechanism $\underline{45}$ and the related

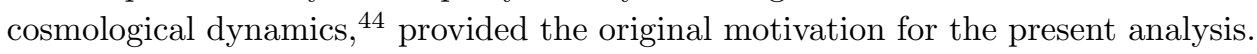
The resulting scenario would associate $\Delta$ to the onset of the inflationary phase. Collecting more information on low multipoles of CMB APS might tell us something more definite about how an inflationary regime was originally attained.

\section{Features in the primordial fluctuations}

The most recent CMB data by the Planck satellite ${ }^{1}$ are in excellent agreement with the assumption of adiabatic primordial scalar perturbation with nearly scaleinvariant PS, described by a simple power law with spectral index $n_{s}$ very close to (albeit different from) unity ${ }^{46}$ It would be produced in the simplest inflationary scenario, in which a single minimally-coupled scalar field slowly rolls down a smooth potential. In spite of this, models that account for localized 'features' in the PPS could provide a better fit to data with respect to a smooth power-law spectrum. These features could be produced in inflationary models with departures from the near-scale-invariant-power-law spectrum of the standard simplest scenario, and observable signatures would be in the CMB anisotropy temperature APS and in the matter PS from galaxy surveys. We analyze here three classes of models, and Fig. 4 displays the corresponding PPS shapes. 
(i) Log-spaced oscillations model assumes an oscillation in proper time affecting the
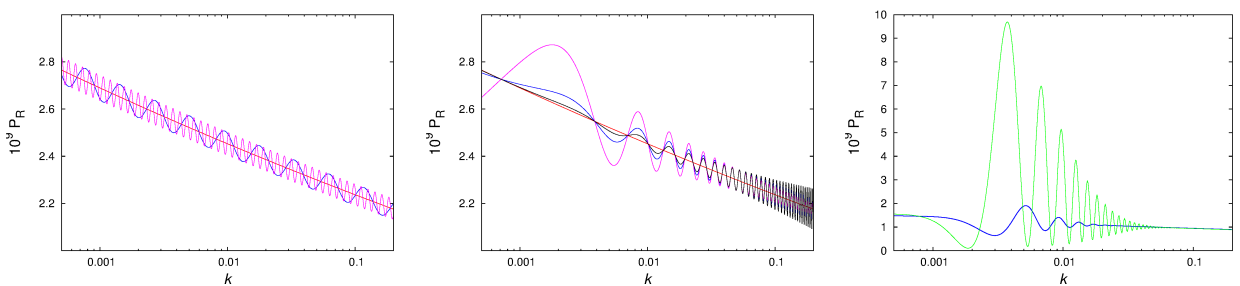

Fig. 4. PPS simulated for various oscillatory models parametrized with an amplitude, a frequency and a phase (for the Linear oscillation models we have the scale-dependence index as further parameter). Left: Log-spaced oscillations models for different values of the frequency oscillation parameter. Middle: Linear oscillation models for different values of the scale-dependence index. Right: Step oscillation models for different values of the frequency and phase parameters.

amplitude of curvature perturbation during the inflationary expansion (producing features periodic in $\ln k$ ). It is the case of models with no-Bunch-Davies initial condition,, 47 or in the bouncing inflationary scenarie $\frac{48}{48}$ or also in the axion monodromy

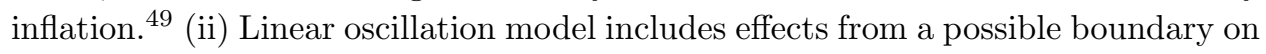
effective field theory (where we assume new physics which occurs at one moment in time, such as a discontinuity in single-field inflation ${ }^{50}$ or a sharp turn in multi-field

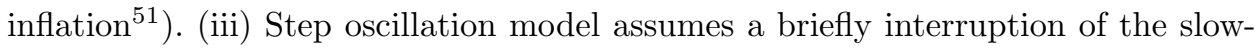
roll. For instance it can happen by a phase transition, a burst of resonant particle production, a sudden turn in field space or a step in the inflation potential. ${ }^{[52}$ It is found to be essentially a power-law with superimposed oscillations localized only in a limited range of wavenumbers. It is noteworthy that this kind of models is able to produce oscillation at very high- $\ell$, and it is very interesting looking to the CMB temperature anisotropies glitches in correspondence of $\ell=22$ and $\ell=40$, first observed by the WMAP experiment and later confirmed by the Planck satellite.

We used here the Planck 2013 data release and a combined CMB and SDSS (DR-11) ${ }^{53}$ dataset. Our results show that using the CMB data alone we have noevidence of improving the concordance with data and agree with the more recent results of the Planck Collaboration ${ }^{46}$ Instead, using the combined dataset of CMB SDSS-DR11 data, we can see a positive Bayesian evidence for the inflationary logspaced oscillation and step oscillation models. Updating this analysis with final Planck data will be very interesting to confirm or discard these kinds of models.

\section{Primordial non-Gaussianity}

The study of primordial non-Gaussianity (PNG) provides a powerful tool to shed light on early Universe mechanisms for the generation of primordial perturbations (see e.g. Refs. [54, 55] and references therein). Different inflationary models predict different amplitudes, shapes, and scale dependence of PNG. As a result, PNG allows 
to discriminate between models that can show degeneracies considering only the APS.

One of the main goals of these analyses is to constrain the amplitude and shape of PNG using the angular bispectrum of CMB anisotropies, related via linear radiation transfer to the primordial bispectrum, $B_{\Phi}\left(k_{1}, k_{2}, k_{3}\right)$, defined by

$$
\left\langle\Phi\left(\vec{k}_{1}\right) \Phi\left(\vec{k}_{2}\right) \Phi\left(\vec{k}_{3}\right)\right\rangle=(2 \pi)^{3} \delta^{(3)}\left(\vec{k}_{1}+\vec{k}_{2}+\vec{k}_{3}\right) B_{\Phi}\left(k_{1}, k_{2}, k_{3}\right) ;
$$

here $\Phi$ is the primordial potential defined in terms of the comoving curvature perturbation $\zeta$ on super-horizon scales by $\Phi \equiv(3 / 5) \zeta$. The bispectrum measures the correlation among three perturbation modes, and it is expected to be zero for Gaussian perturbations. In general, the bispectrum can be written as

$$
B_{\Phi}\left(k_{1}, k_{2}, k_{3}\right)=f_{\mathrm{NL}} F\left(k_{1}, k_{2}, k_{3}\right),
$$

where $f_{\mathrm{NL}}$ is the so-called "nonlinearity parameter", a dimensionless parameter measuring the amplitude of non-Gaussianity. The functional dependence of $F\left(k_{1}, k_{2}, k_{3}\right)$ on the type of triangle formed by $k_{1}, k_{2}, k_{3}$ defines the shape of the bispectrum. $\frac{[56}{5}$ Even in the simplest models of inflation, consisting of a single slowly-rolling scalar

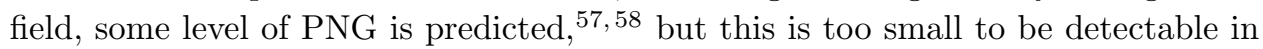
CMB and LSS surveys. Large level of PNG can be produced however in multi-field scenarios, or in single-field models with non-standard Lagrangians and deviations from Bunch-Davies vacuum, and in many other cases. Each of the scenarios outlined above predicts different shapes, the main of which are briefly described below.

Local shape, where the signal peaks in "squeezed" triangles $\left(k_{1} \ll k_{2} \simeq k_{3}\right)$. This shape is typically generated in multi-field models of inflation. Equilateral shape, peaking on equilateral bispectrum triangles $\left(k_{1} \approx k_{2} \approx k_{3}\right)$. Examples of this class include single-field models with non-canonical kinetic term, 59 such as e.g. Dirac-BornInfeld (DBI) inflation,$\frac{60}{6}$ models characterized by more general higher-derivative interactions of the inflaton field, and models arising from effective field theories $\frac{61}{61}$ Folded (flattened) shape, peaking on isosceles, nearly degenerate triangles. Examples of this class include e.g. single-field models with non-Bunch-Davies vacuum. Orthogonal shape, which is generated, e.g., in single-field models of inflation with a non-canonical kinetic term, or with general higher-derivative interactions. Notably, the folded shape described above can be obtained as a linear combination of equilateral and orthogonal shapes. In light of this, actual measurements of PNG generally focus on local, equilateral and orthogonal templates.

It must be noted that many but not all models are included in the previous classification. For example, models with a temporary breaking of slow-roll conditions generate strongly scale-dependent, oscillatory shapes that cannot be approximated by a combination of local, equilateral and orthogonal templates. Given the limited scope of this review, the focus here will be however only on the above main shapes.

Having computed the CMB angular bispectrum templates arising from the various primordial shapes, non-Gaussianity estimation consists then essentially in fitting such templates to the 3-point function of the data in order to measure the 
best-fit amplitude parameters $f_{\mathrm{NL}}$. This apparently straightforward approach actually presents many statistical and numerical complications. These arise mainly from the huge number of modes (triangles) which contribute to the signal for high resolution experiments like Planck and WMAP, and from spurious mode couplings from sky-cut and anisotropic instrumental noise. Several numerical techniques have been successfully tested in the literature and implemented by the Planck team in order to address these issues. This gave rise to several independent, but ultimately equivalent, Planck bispectrum analysis pipelines, the so called modal, binned and separable template-fitting estimators, that were separately applied to the data 62

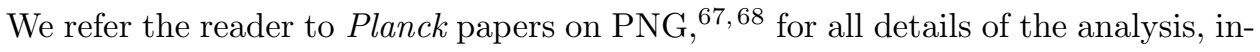
cluding validation tests carried on data and simulations, descriptions of the various pipelines, and constraints on a much larger set of shapes than the three discussed here.

The final Planck results for the local, equilateral and orthogonal shapes, from the 2015 combined analysis of temperature and polarization data, are as follows:

$$
f_{\mathrm{NL}}^{\text {local }}=+0.8 \pm 5.0 ; \quad f_{\mathrm{NL}}^{\text {equil. }}=-4 \pm 43 ; \quad f_{\mathrm{NL}}^{\text {ortho. }}=-26 \pm 21 .
$$

The main conclusion from Planck is that consistency with Gaussianity is found in all cases (including shapes not considered here). Planck bispectrum constraints lead to important implications for inflationary model building, such as a lower bound on the sound speed in effective single field inflation theory, or limits on the curvaton decay fraction, and so on. In light of the current results, the simplest slowroll single field inflationary paradigm has passed its most stringent and accurate test to date (although alternative, more complex, possibilities, while constrained, are by no means ruled out yet). Planck has extracted nearly all the possible PNG information from CMB data. Even an ideal, noiseless temperature+polarization experiment would improve on current error bars by at most a factor 2. For substantial improvements it will be necessary to look at different observables and wait for future experiments. LSS could be in principle promising, since it contains more modes than the CMB. Precise primordial bispectrum estimation from LSS surveys is however very hard due to non-linearities from gravitational evolution, galaxy-bias and other effects; whether we will be able to achieve improvements from the LSS bispectrum will strongly depend on how well we can keep all these systematics under control, and it is at present an open question. Two-point function based measurements of large scale signatures arising from scale-dependent halo bias look on the other hand quite promising, and have the potential of achieving $f_{\mathrm{NL}} \sim 1$ sensitivity for the local shape ${ }^{69}$ It has been pointed out that the study of the bispectrum of $21 \mathrm{~cm}$ radiation or measurements of cross-correlations between CMB spectral distortions and temperature anisotropies can in principle improve over current bounds by more than one order of magnitude. These are fascinating but futuristic prospects, since highsensitivity $f_{\mathrm{NL}}$ measurements with these techniques will require either full-sky 21 cm surveys with redshift tomography in the $30 \lesssim z \lesssim 100$ range, or high-resolution maps of angular anisotropies of the CMB $\mu$-distortion parameter 70 71 


\section{Limits on neutrino masses from cosmology and particle physics}

The absolute scale of neutrino masses is one of the main open issues both in cosmology and particle physics. Current experimental strategies involve i) measurements exploiting kinematics effects in beta decay: ${ }^{72}$ ii) searches for neutrinoless double beta decay (' $0 \nu 2 \beta$ '),$\frac{73}{3}$ and iii) cosmological observations ${ }^{74}$ The three approaches are complementary, each of them presenting its own advantages and disadvantages and being sensitive to slightly different quantities related to the neutrino masses ${ }^{75}$ In this work, we derive joint constraints on neutrino mass parameters from the most recent observations from both laboratory and cosmological experiments, and forecasts, combining them in the framework of Bayesian statistics. In particular, for ' $0 \nu 2 \beta$ ' experiments, we take into account the uncertainty related to nuclear matrix elements, in order to account its impact on the neutrino mass estimates.

\subsection{Neutrino parameters, method and data}

We denote the masses of the neutrino mass eigenstates $\nu_{i}$ with $m_{i}(i=1,2,3)$. $\Delta m_{21}^{2}$ represents the difference between the two eigenstates closest in mass, while the sign of $\Delta m_{31}^{2}$ discriminates between the normal (NH, $\left.\Delta m_{31}^{2}>0\right)$ and inverted $\left(\mathrm{IH}, \Delta m_{31}^{2}<0\right)$ hierarchies. The neutrino mass eigenstates are related to the flavour eigenstates $\nu_{\alpha}(\alpha=e, \mu, \tau)$ through $\nu_{\alpha}=\sum_{i} U_{\alpha i} \nu_{i}$, where $U_{\alpha i}$ are the elements of the neutrino mixing matrix $U$, parameterized by the three mixing angles $\left(\theta_{12}, \theta_{23}, \theta_{13}\right)$, one Dirac $(\delta)$ and two Majorana $\left(\alpha_{21}, \alpha_{31}\right) \mathrm{CP}$-violating phases. Oscillation phenomena are insensitive to the Majorana phases, that however affect $0 \nu 2 \beta$ decay. The different combinations of the mass eigenvalues and of the elements of the mixing matrix probed by the experimental avenues are: the squared effective electron neutrino mass $m_{\beta}^{2} \equiv \sum_{i}\left|U_{e i}\right|^{2} m_{i}^{2}$ ( $\beta$ decay experiments), the effective Majorana mass $m_{\beta \beta} \equiv\left|\sum_{i} U_{e i}^{2} m_{i}\right|(0 \nu 2 \beta$ searches $)$, the sum of neutrino masses $M_{\nu} \equiv \sum_{i=1}^{3} m_{i}$ (cosmological observations). We perform a Bayesian analysis based on a MCMC method, using cosmoMc ${ }^{76}$ as a generic sampler. We consider the following vector of base parameters: $\left(M_{\nu}, \Delta m_{21}^{2}, \Delta m_{31}^{2}, \sin ^{2} \theta_{12}, \sin ^{2} \theta_{13}, \phi_{2}, \phi_{3}, \xi\right)$ where $\phi_{2} \equiv \alpha_{21}, \phi_{3} \equiv \alpha_{31}-2 \delta$ and $\xi$ is a "nuisance" parameter related to the nuclear modeling uncertainty. We assume uniform prior distributions for all parameters and neglect the mixing angle $\theta_{23}$, irrelevant for mass parameters.

Our baseline dataset is the global fit of the updated neutrino oscillation parameters ${ }^{77}$ We model the likelihood as a the product of individual Gaussians (correlations can be neglected $\left.{ }^{77} \mid 78\right)$ d KATRIN $^{\sqrt{79}}$ and HOLMES $S^{80}$ represent our forthcoming and next-generation direct measurement datasets, respectively. We take the likelihood for kinematic measurements to be a Gaussian in $m_{\beta}^{2}>0$, with a width given by the expected sensitivity of the experiment, i.e. $\sigma\left(m_{\beta}^{2}\right)=0.025,0.006 \mathrm{eV}^{2}$ for KATRIN and HOLMES, respectively. For $0 \nu 2 \beta$ searches, we consider the current

\footnotetext{
${ }^{\mathrm{d}}$ See http://www.nu-fit.org/ for results updated after the Neutrino 2014 conference.
} 
data from the GERDA experiment ${ }^{81}$ as the present dataset, its upgrade (GERDAII) for the near-future, and the nEXO experiment ${ }^{e}$ as a next-generation dataset. $0 \nu 2 \beta$ experiments are sensitive to the half-life of $0 \nu 2 \beta$ decay $T_{1 / 2}^{0 \nu}$. If neutrinos are Majorana particles, $T_{1 / 2}^{0 \nu}$ is related to the Majorana effective mass through $T_{1 / 2}^{0 \nu}=\frac{1}{G^{0 \nu}\left|\mathcal{M}^{0 \nu}\right|^{2}} \frac{m_{e}^{2}}{m_{\beta \beta}^{2}}$, where $m_{e}$ is the electron mass, $G^{0 \nu}$ is a phase space factor and $M^{0 \nu}$ is the nuclear matrix element.

We model the likelihood of $0 \nu 2 \beta$ experiments as a Poisson distribution in the number of observed events, with an expected value $\lambda=\lambda_{S}+\lambda_{B}$ given by the sum of signal $(S)$ and background $(B)$ contributions. For a given value of $T_{1 / 2}^{0 \nu}$, the expected number of signal events observed in a time $T_{\text {obs }}$ for a detector mass $M$ is $\lambda_{S}=\frac{\ln 2 N_{A} \mathcal{E} \epsilon}{m_{e n r} T_{1 / 2}^{0 \nu}}$ where $N_{A}$ is Avogadro's number, $\mathcal{E} \equiv M T_{\mathrm{obs}}$ is the exposure, $\epsilon$ is the detector efficiency, $m_{e n r}$ is the molar mass of the enriched element involved in the decay. The level of background is given by the "background index", i.e. the number of expected background events per unit mass and time within an energy bin of unit width. For GERDA-I, we use the parameters reported in Tab. I of Ref. 81 for the case with pulse-shape discrimination. For GERDA-II, we consider a reduction of the background index down to $10^{-3}$ counts $\mathrm{keV}^{-1} \mathrm{~kg}^{-1} \mathrm{yr}^{-1}$, a total exposure of $120 \mathrm{~kg}$ yr, and the same efficiency as GERDA-I ${ }^{82}$ For nEXO, we assume a background index corresponding to 3.7 events ton ${ }^{-1} \mathrm{yr}^{-1}$ in the region of interest and an exposure of 25 ton yr,, 83 and the same efficiency as EXO $\underline{84}$

In order to account for the uncertainty related to nuclear modeling, 855 we compute $T_{1 / 2}^{0 \nu}$ for a given $m_{\beta \beta}$ using fiducial values of nuclear matrix elements (NME) and axial coupling constant, and then rescale it by a factor $\xi^{2}$ (see Ref. [86] for a similar approach). For what concerns the cosmological dataset, we use the posterior distribution of $M_{\nu}$ from the combination of Planck temperature and polarization data with baryon acoustic oscillations (BAO) $\frac{1}{1}$ as both our current and forthcoming reference dataset. Finally, we consider the Euclid mission (weak lensing tomography, galaxy clustering and ISW) in combination with data from Planck ${ }^{21}$ as our reference next-generation experiment. We model the likelihood as Gaussian in $M_{\nu}=0.1 \mathrm{eV}$, with $\sigma\left(M_{\nu}\right)=0.06 \mathrm{eV}$ and the addition of the physical prior $M_{\nu}>0$.

\subsection{Results}

We present our results for $M_{\nu}, m_{\beta}$ and $m_{\beta \beta}$ in Fig. 5 both in the case where $\xi$ is fixed to 1 and when $\xi$ is marginalized over, in order to show the impact of uncertainties in nuclear modeling. Notice that the low mass region is excluded by the oscillation data, with the only exception of $m_{\beta \beta}$ in the case of $\mathrm{NH}$; the reason is that in this case the phases can arrange in order to yield $m_{\beta \beta}=0$ even for finite values of the mass differences. Similar limits are provided by the "present" dataset independently of whether nuclear uncertainties are marginalized over: present con-

$\overline{\text { e }_{\text {https://www-project.slac.stanford.edu/exo/ }}}$ 

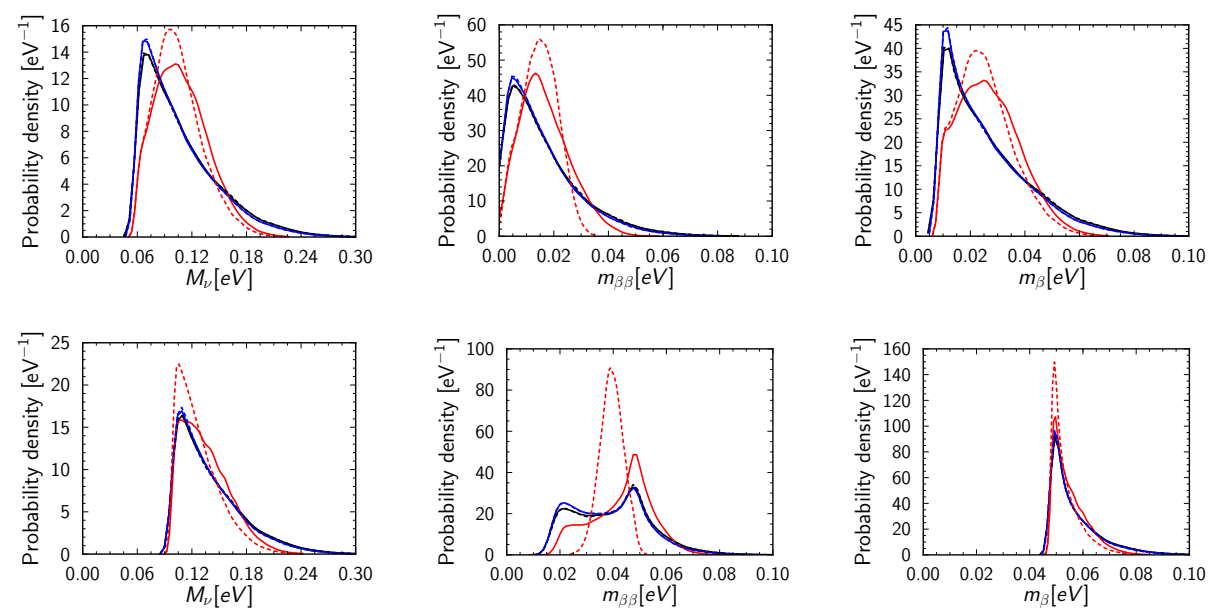

Fig. 5. Posterior distributions for the neutrino mass parameters, for NH (top row) and IH (bottom row). Solid (dashed) curves correspond to marginalization over nuclear uncertainties (fixed fiducial values for nuclear parameters). Black, blue and red curves refer to present, forthcoming and nextgeneration datasets, respectively.

straints are dominated by the cosmological limit on $M_{\nu}$, that translates directly to bounds on $m_{\beta}$ and $m_{\beta \beta}$ once oscillation data are taken into account. Forthcoming datasets yield similar constraints for the mass parameters: the upgraded sensitivity of GERDA-II and the inclusion of KATRIN provide a marginal improvement to the Planck+BAO plus oscillations data combination. Substantial differences arise for next-generation experiments. In this case, cosmological observations and $0 \nu 2 \beta$ searches have comparable constraining power, and the nuclear uncertainties have a dramatic impact in deriving parameter constraints. Marginal evidence for nonminimal mass parameters can be highlighted in the case of normal hierarchy, even when nuclear uncertainties are taken into account.

The combination of current and forthcoming data from oscillation, kinematic, $0 \nu 2 \beta$ and cosmological experiments allows to put upper bounds on the neutrino mass parameters. Since these limits are dominated by the combination of oscillations and cosmological data, they are not affected by uncertainties in nuclear modeling. For $M_{\nu}=0.1 \mathrm{eV}$ and a factor 2 uncertainty in nuclear modeling, future experiments will ideally allow to measure non-minimal mass parameters with a $95 \%$ accuracy.

\subsection{Limits on neutrino masses in a non-standard PPS scenario}

In order to study how the cosmological constraints on the parameters change in more general inflationary scenarios, we assume a non-parametric form for the PPS. In particular, we decide to parametrize the scalar PPS with a piecewise cubic Hermite interpolating polinomia ${ }^{87}$ (PCHIP) to avoid some unwanted oscillating behaviour related to the natural cubic spline function (see Appendix A of Ref. [88). We consider a $\Lambda \mathrm{CDM}$ model with three degenerate active massive neutrinos together 
with the PPS approach. We also explore a scenario with three active light massive neutrinos plus one massive sterile neutrino species characterized by an effective mass $m_{s}^{\text {eff }}$.

Our baseline data set consists of the Planck 2015 satellite CMB temperature and polarization APS 1089 We also consider a prior on the Hubble constant, $H_{0}$, estimated from a reanalysis of Cepheids data ${ }^{90}$ and include measurements of the LSS in the form of BAO. In particular, we use the 6dFGS, SDSS-MGS and BOSS DR11 measurements 91

\begin{tabular}{c|ccc}
\hline \hline $\begin{array}{c}\text { Extended } \\
\text { Model }\end{array}$ & $\begin{array}{c}\text { Planck } \\
\text { Pol }\end{array}$ & $\begin{array}{c}\text { Planck } \\
\text { Pol }\end{array}$ & $\begin{array}{c}\text { Planck } \\
\text { Pol } \\
\Lambda \mathrm{CDM}+\end{array}$ \\
\hline$m_{\nu}$ & $<0.80$ & $<0.20$ & $<0.43$ \\
\hline$\Sigma m_{\nu}$ & $<0.68$ & $<0.21$ & $<0.44$ \\
$N_{\text {eff }}$ & $<3.65$ & $<3.52$ & $<3.68$ \\
$m_{s}^{\text {eff }}$ & $<1.13$ & $<0.61$ & $<1.06$ \\
\hline \hline
\end{tabular}

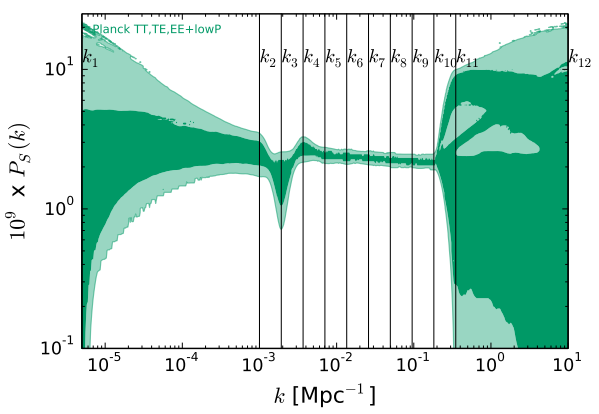

Fig. 6. Left: 95\% CL on the active (sterile) neutrinos masses and on the total massive neutrino species, $N_{\text {eff }}$, from the combination of considered data sets. Right: $68 \%, 95 \%$ and $99 \%$ CL allowed regions for the PCHIP PPS scale dependence in the $\Lambda \mathrm{CDM}+\sum m_{\nu}$ model, using CMB data only.

The results are shown in table in the left panel of Fig. 6. In the first scenario, concerning only CMB measurements, the bound on the sum of massive neutrinos is largely relaxed with respect to the the power-law model $\left(\sum m_{\nu}<0.49 \mathrm{eV}\right.$ at 95\% CL) 1 In the second scenario, there is no evidence for neutrino masses nor for non-zero sterile neutrino mass. Concerning only CMB measurements, the bound on the sum of massive neutrinos is more stringent with respect to previous scenario. The reason for that is due to the degeneracy between $\sum m_{\nu}$ and $m_{s}^{\text {eff. Notice that }}$ in both scenarios the addition of a prior on the Hubble constant and of the BAO data displaces the bounds on $\sum m_{\nu}$ to lower values in agreement with the standard power-law PPS case ${ }^{1}$ An example of the reconstructed PPS is given in Fig. 6 (right panel). Note that both $P_{s, 1}$ and $P_{s, 12}$ are poorly constrained because of the absence of measurements at their corresponding wavenumbers. All the remaining $P_{s, j}$, with $j=2, \ldots, 11$ are well-constrained. In particular, in the range between $k_{5}$ and $k_{10}$ the PPS can be perfectly described by a power-law parametrization. Moreover we can notice that there is a significant dip at wavenumbers around $k=0.002 \mathrm{Mpc}^{-1}$, that comes from the dip at $\ell=20-30$ in the CMB temperature APS and a small bump around $k=0.0035 \mathrm{Mpc}^{-1}$, corresponding to the increase at $\ell \simeq 40$. 


\section{Robustness of cosmological thermal axion mass bounds}

Relativistic axions contribute to the dark radiation content of the Universe, increasing the effective number of relativistic degrees of freedom $N_{\text {eff }}$ (see Ref. 994 for details), while massive thermal axions, when become non-relativistic, affect the LSS formation suppressing the small scale power, clustering only at scales larger than their free-streaming scale. Massive axions affect also the CMB temperature anisotropies via the early ISW effect. All the cosmological axion mass limitsf assumed the usual simple power-law description for the primordial perturbations, defined by an amplitude and a scalar spectral index. In Ref. 94] the thermal axion mass is constrained using a non-parametric description of the scalar perturbation PPS, to test the robustness of its bounds. We adopted a function, the PCHIP97 in the same modified version $\sqrt{88}$ as in Sect. 6.3 , to interpolate the PPS values in a series of nodes at fixed position.

We discuss here the $\Lambda \mathrm{CDM}$ model, extended with the axions hot thermal relics, together with the PPS approach (see Ref. 94 for a similar analysis with two coexisting hot dark matter species, thermal axion and massive neutrinos). We consider various CMB measurements: the temperature data from the Planck satellite 98 the WMAP-9yrs polarization measurements, $\frac{100}{1}$ the SPT ${ }^{101}$ and $\mathrm{ACT}^{21}$ datasets.

Concerning CMB datasets only, the bounds on the thermal axion mass are unconstrained in the case in which the PPS is not described by a simple power-law (see Tab. III in Ref. 94), while in this last case $m_{a}<1.83 \mathrm{eV}$ (see Tab. IV in Ref. 94). Including the Hubble Space Telescope (HST) prior on the Hubble constant, ${ }^{90}$ $H_{0}=70.6 \pm 3.3 \mathrm{~km} / \mathrm{s} / \mathrm{Mpc}$, provides a $95 \% \mathrm{CL}$ upper limit on the thermal axion mass of $1.31 \mathrm{eV}$. The further addition of the BAO measurements 91$]$ 93 102$] 105$ brings this constraint down to $0.91 \mathrm{eV}$, being these last data sets directly sensitive to the thermal axion free-streaming nature. These upper bounds are very similar to the ones obtained considering the standard power-law PPS (see Tab. IV in Ref. 94]). Adding the CFHTLenS ${ }^{106}$ bounds on the $\sigma_{8}-\Omega_{m}$ relationship, the thermal axion mass bounds become weaker $m_{a}<1.29 \mathrm{eV}$, since this dataset prefers a lower $\sigma_{8}$ value. Finally, considering the Planck Sunyaev-Zeldovich (PSZ) 2013 catalogue ${ }^{107}$ dataset with fixed cluster mass bias, $\sigma_{8}\left(\Omega_{m} / 0.27\right)^{0.3}=0.78 \pm 0.01$, together with the CMB, BAO and HST measurements, a non-zero thermal axion mass of $\sim 1 \mathrm{eV}$ is favored at $\sim 4 \sigma$ level. Using more realistic approaches for the cluster mass bias, $\frac{107}{10}$ $\sigma_{8}\left(\Omega_{m} / 0.27\right)^{0.3}=0.764 \pm 0.025$, the errors on the so-called cluster normalization condition are larger, and, consequently, the preference for a non-zero axion mass is reduced.

Our results are summarized in Fig. 7. In conclusion, using a non-parametric description $[88$ of the scalar perturbation PPS that relaxes the power-law assumption in Ref. [94, we tested the robustness of the cosmological axion mass bounds, found to be only mildly sensitive to the PPS choice and therefore not strongly dependent

\footnotetext{
${ }^{\text {f }}$ See e.g. Refs. 95 96 for recent cosmological constraints on thermal and non-thermal axions.
} 
on the particular details of the underlying inflationary model.
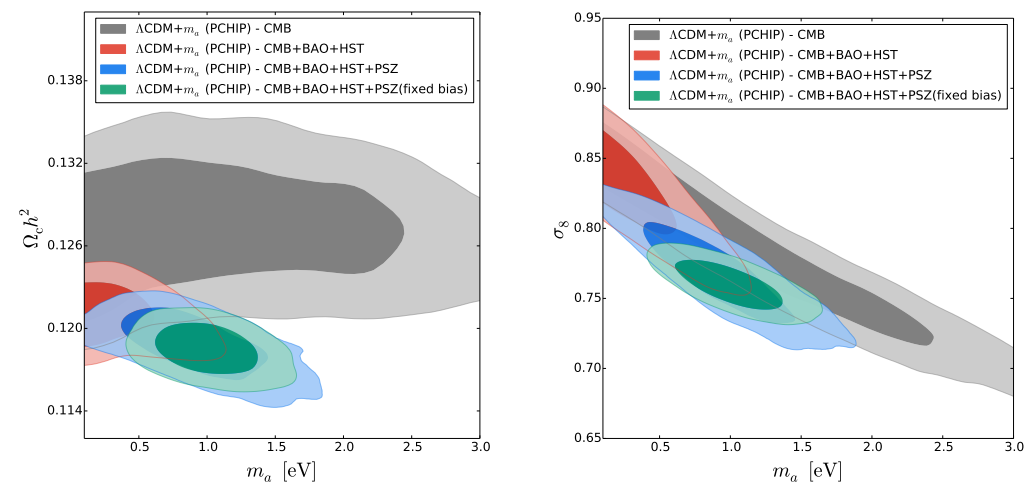

Fig. 7. $68 \%$ and $95 \%$ CL allowed regions in the $\left(m_{a}, \Omega_{c} h^{2}\right)$ plane (left panel) and in the ( $m_{a}$, $\sigma_{8}$ ) plane (right panel) for different data combinations, when a PCHIP PPS is assumed. From Ref. [94].

\section{Cosmological constraints on the neutron lifetime}

The study of the neutron lifetime, $\tau_{n}$, a fundamental quantity in nuclear physics, is fascinating since the current status of particle physics experiments is still puzzling and unclear. The current used value is the one quoted by the Particle Data Group ${ }^{108} \tau_{n}=(880.3 \pm 1.1) \mathrm{s}$ and it is obtained as an average between the seven most recent experiments, bottle-method and beam-method like (for further details see [109]). Combining the five most recent bottle-method measurements one obtain the tight constraint $\tau_{n}=(879.6 \pm 0.8) \mathrm{s}$ while from the two most recent beammethod measurements one obtain $\tau_{n}=(888.0 \pm 2.1) \mathrm{s}$. Given this tension, it is interesting to investigate if cosmological measurements can constrain the neutron lifetime in an independent way with respect to particle physics experiments, thus testing them, and, moreover, to address the implications for cosmology of a a more precise determination of the neutron lifetime.

We start discussing constraints on $\tau_{n}$ from current cosmological data. Assuming Standard Big Bang Nucleosynthesis it is possible to evaluate primordial abundances of light elements from CMB as functions of few parameters: ${ }^{110}$ the baryonic abundance, the relativistic degrees of freedom, the chemical potential of electron neutrinos and the neutron lifetime. Neglecting the chemical potential, considering the high precision achieved in the determination of baryonic abundance and fixing $N_{\text {eff }}$ to its standard value of 3.046, from primordial abundances (in particular Helium abundance) we can infer the value of the neutron lifetime. We start analyzing Planck 2015 results as CMB dataset with the publicly available MCMC package cosmoMC. Table 1 reports the most interesting results (for complete analysis see Ref. [111).

The next step is to combine CMB observations with direct astrophysical measurements of Helium. We consider eight primordial Helium measurements collected 
in the last ten years and combine them with Planck data and select two possible independent combinations of these astrophysical datasets (referred to as M12-P for Refs. [112, 113] and M12-I14 for Refs. [112, 114]). As shown in Table 1, combining the constraining power of CMB data, sensitive to the baryon density, with the Helium astrophysical measurements we obtain more stringent limits on the neutron lifetime, with respect to cosmological data only.

We extend the analysis performing some forecasts on future cosmological experiments. Considering that CMB sensitivity on $\tau_{n}$ is encoded in the small-scale region, we expect tighter constraints from next CMB projects planned to measure the high $\ell$ range. As reported in Table 1 the most stringent constraint is obtained by the combination of future experiments COrEg and Euclid, giving $\tau_{n}=(880.3 \pm 6.7) \mathrm{s}$.

Table 1. Values of $\tau_{n}$ with $1 \sigma$ erros for cosmological and astrophysical datasets.

\begin{tabular}{|c|c|}
\hline Dataset & $\tau_{\mathbf{n}}[\mathrm{s}]$ \\
\hline \hline Planck + BAO + Lensing & $894 \pm 63$ \\
\hline \hline M12-I14 & $905.7 \pm 7.8$ \\
M12-P & $886.7 \pm 8.8$ \\
\hline \hline COrE & $880 \pm 11$ \\
CVL & $880.7 \pm 5.5$ \\
COrE + Euclid & $880.3 \pm 6.7$ \\
\hline
\end{tabular}

In conclusion, the combination of CMB anisotropies and astrophysical observations allows to obtain stringent limits and shed light on the present experimental discrepancies, while future cosmological missions, such as COrE and Euclid, could reach a sensitivity comparable with that of current experiments.

\section{Testing general relativity with cosmic polarization rotation}

The CPR provides a test of the EEP, which is the foundation of any metric theory of gravity, including GR. Almost all the information about the Universe outside the solar system is carried to us by photons, with their direction, energy and polarization. The latter consists essentially in the position angle (PA) of the polarization ellipse, i.e. photons carry throughout the Universe an important geometrical information. To properly use this information, it is important to know if and how it is changed while photons travel to us. The directions of photons can be modified by gravitational fields and their energies are modified by the Universe expansion, while the polarization PA is modified while photons travel in a plasma with a magnetic field, the so called Faraday rotation, proportional to the wavelength squared. Is

ghttp://www.core-mission.org/ 
the polarization PA also modified while photons travel large distances in vacuum? Searches for CPR deal with this important question.

Clearly, if the CPR angle $\alpha$ is not zero, it should be either positive for a counterclockwise rotation, or negative for a clockwise rotation (we adopt the IAU convention 115 for PA, which increases counter-clockwise facing the source, from North through East). Therefore symmetry must be broken at some level, leading to the violation of fundamental physical principles (see Ref. [116] for a recent review). Indeed CPR is linked also to a possible violation of the EEP. The reasons for the link are due to the unique counterexample to Schiff's conjecture ${ }^{117}$ that any consistent Lorentz-invariant theory of gravity which obeys the weak equivalence principle (WEP) would also obey the EEP, which involves a pseudoscalar field, producing CPR 118 Therefore, if we could show that $\alpha=0^{\circ}$, the EEP would be tested with the same high accuracy of the WEP, greatly increasing our confidence in the EEP and then in GR. See Ref. [119] for a recent review of CPR tests.

CPR tests are simple in principle: they require a distant source of polarized radiation with established polarization orientation at the emission, $P A_{e m}$. By measuring the observed orientation $P A_{o b s}$, the CPR angle can be calculated:

$$
\alpha=P A_{o b s}-P A_{e m} .
$$

The problem is the estimate of $P A_{e m}$. Fortunately it can be solved using the fact that scattered radiation is polarized perpendicularly to the plane containing the incident and scattered rays. This simple physical law has been applied to CPR tests, using both the ultraviolet (UV) radiation of radio galaxies (RG) and the tiny anisotropies of the CMB. The first CPR tests 25 years ago used instead a statistical analysis of the radio polarization in RG ${ }^{120}$ The most accurate CPR tests obtained with the various methods are summarized in Fig. 8, based on data in Ref. 119.

In summary, the results so far are consistent with a null CPR with upper limits of the order of one degree.

\subsection{Current problems and future prospects}

Searches for CPR using the UV polarization of RG have reached the limits allowed by current instrumentation, for the lack of RG suitable for the test and bright enough so that their polarization can be measured with the available instruments.

The most accurate results are now obtained with the CMB polarization, averaging over large sky areas, i.e. assuming uniform CPR over these areas. A current problem with CPR searches using the CMB is the calibration of the polarization PA for the lack of sources with precisely known PA at CMB frequencies. This introduces a systematic error, similar to the statistical measurement error, of about $1^{\circ}$ (see Fig. 8). Recently, the polarization PA of the Crab Nebula (Tau $\alpha$ ) has been measured with an accuracy of $0.2^{\circ}$ at $89.2 \mathrm{GHz}{ }^{121}$ However, most CMB polarization measurements are made at $100-150 \mathrm{GHz}$ and the Crab Nebula is not visible from the South Pole, the site of several CMB experiments. In order to overcome 


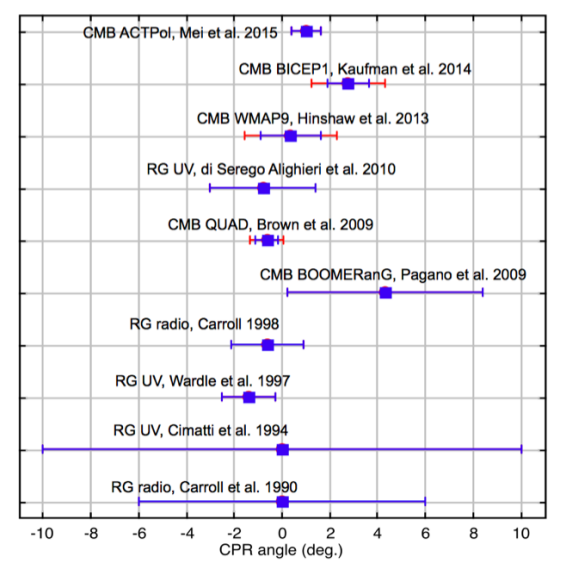

Fig. 8. CPR angle measurements by the various experiments, displayed in chronological order. Blue error bars: statistical errors; red error bars: including also systematics, if present/available. A systematic error should be added to the ATCPol measurement, equal to the unknown difference of the Crab Nebula polarization PA between $146 \mathrm{GHz}$ and $89 \mathrm{GHz}$.

the PA calibration problem, some CMB polarization experiments have used a $T B$ and $E B$ nulling procedure ${ }^{1222}$ but this would eliminate together the PA systematic error and any CPR angle $\alpha$, so it cannot be used for CPR tests.

Furthermore, we note that, unfortunately, in the pixelization too ${ }^{123}$ widely adopted in CMB experiments $\mathrm{h}^{\mathrm{h}}$ the polarization PA is assumed to increase clockwisely (looking at the source), which is opposite to the standard IAU convention, adopted in other bands, thus calling for caution when comparing measures with different methods, like for CPR tests.

The different methods are complementary in many ways. They cover different wavelength ranges and the methods at shorter wavelength have an advantage, if CPR effects grow with photon energy, as foreseen in some cases $\frac{125] 126}{126}$ They also reach different distances, and the CMB method obviously reaches furthest. However the relative difference in light travel time between $\mathrm{z}=3$ and $\mathrm{z}=1100$ is only $16 \%$.

Improvements are expected by better targeted high resolution radio polarization measurements of RGs and quasars, by more accurate UV polarization measurements of RGs with the coming generation of giant optical telescopes, 127-129 and by future CMB polarization measurements such as those from Planck ${ }^{27}$ and BICEP3 ${ }^{130}$ Indeed, the Planck satellite has a very low statistical error $\left(\sim 0.06^{\circ}\right)$ for CPR measurements, but to exploit its great accuracy a significant reduction in the systematic error in the calibration of the polarization angle (currently of $\sim 1^{\circ}$ for CMB polarization experiments) is needed (see also Ref. [131]). Great opportunities will come from more precise polarization measurements of celestial sources at CMB frequencies with ATCA ${ }^{[132}$ and ALMA,,$\frac{133}{13}$ and by a calibration source on a satellite. ${ }^{134}$

${ }^{\mathrm{h}}$ See Ref. 124 for a pixelization software adopting IAU convention. 


\section{SKA contribution to future CMB spectrum experiments}

Recent limits on $\mathrm{CMB}$ spectral distortions and constraints on energy dissipation processes in the plasma $2^{135}$ are mainly set by COBE/FIRAS experiment ${ }^{136}$ High accuracy CMB spectrum space experiments, such DIMES $(\lambda \gtrsim 1 \mathrm{~cm})$ and FIRAS II $(\lambda \lesssim 1 \mathrm{~cm})$, were proposed to constrain energy exchanges up to 100 times better than FIRAS. Dissipation processes at early times $\left(z \gtrsim 10^{5}\right)$ result in Bose-Einstein (BE)-like distortions, $\frac{137}{137}$ while late epochs mechanisms $\left(z \lesssim 10^{4}\right)$ before or after the recombination era generate Comptonization and free-free (FF) distortions. 138 New space missions were proposed to probe cosmic origin and evolution observing CMB temperature and polarization anisotropies with $\sim$ degree resolution, as

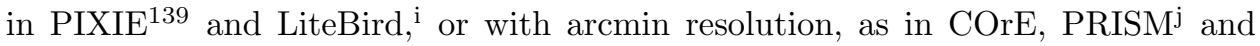
COrE + , in combination with spectrum measurements in the case of PIXIE and PRISM. SKA extremely high sensitivity and resolution can contribute to set new constraints on CMB spectral distortions beyond current limits. Improved absolute temperature measures will strengthen the constraints on CMB spectrum affected by (pre-recombination) decaying and annihilating particles, by superconducting cosmic strings electromagnetic radiation, by energy injection of evaporating primordial black holes $(\mathrm{BH})$. Spectral distortions could constrain non evaporating $\mathrm{BH}$ spin, small scale magnetic fields, vacuum energy density decay, axions. In general, departure of CMB spectrum from a perfect blackbody is theoretically predicted by! $140(i)$ cosmological reionization, producing electron heating and physically correlated Comptonization distortion (with typical Comptonization parameter $y \simeq(1 / 4) \Delta \varepsilon / \varepsilon_{i} \approx 10^{-7}-10^{-6}$ proportional to the fractional energy exchanged in the interaction), and free-free ( $\mathrm{FF}$ ) distortion; (ii) dissipation of primordial perturbations at small scales, damped by photon diffusion and thus invisible in CMB anisotropies, produces BE-like distorted spectra characterized by a positive chemical potential $\mu_{0} \simeq 1.4 \Delta \varepsilon / \varepsilon_{i} \approx 10^{-9}-10^{-7}$; (iii) BE condensation of CMB photons by colder electrons associated with the matter temperature decrease in the expanding Universe relatively faster than that of radiation gives $\mu_{0} \approx-3 \times 10^{-9}$. The above FF signal is the most relevant type of low-frequency global spectral distortion (see Fig. 91. Indeed, the FF term is proportional to the square of baryon density and the structure formation process implies a rate amplification by a factor $\simeq 1+\sigma^{2}$ (being $\sigma^{2}$ the matter distribution variance) with respect to the case of homogeneous plasma ${ }^{142}$ SKA high sensitivity and resolution can also be used to model the contribution from Galactic emissions and extragalactic foreground, a fundamental step to accurately observe these kinds of distortions. Extragalactic source contribution is small compared to Galactic radio emission, currently the major astrophysical problem in CMB spectrum experiments, but, unlike the Galactic emission, it cannot be subtracted from the CMB monopole temperature by exploiting its angular corre-

ihttp://litebird.jp/eng/

${ }_{\mathrm{j}} \mathrm{http}: / /$ www.prism-mission.org/ 
lation properties. A direct radio background estimate from precise number counts will certainly improve the robustness of this kind of analyses. Exploiting the recent differential number counts at $0.153 \mathrm{GHz}, 0.325 \mathrm{GHz}, 1.4 \mathrm{GHz}$, and $1.75 \mathrm{GHz}$ it is possible to evaluate the contribution, $T_{b}$, to the radio background from extragalactic sources in various ranges of flux densities. These signals can be significant at the accuracy level potentially achievable with future experiments. Subtracting sources brighter than several tens of nJy, $T_{b} \lesssim 1 \mathrm{mK}$ at $\nu \gtrsim 1 \mathrm{GHz}$, but $T_{b} \gtrsim 10 \mathrm{mK}$ below $0.3 \mathrm{GHz}$. The minimum source detection threshold is given by the source confusion noise. The finite angular extension of faint galaxies, $\theta \sim 1$ ", implies a "natural confusion limit" $\sim 10 \mathrm{nJy}$ at $\nu \sim 1.4 \mathrm{GHz}$, not a relevant limitation for deep surveys. ${ }^{143}$ At $1 \mathrm{GHz} \lesssim \nu \lesssim$ some $\mathrm{GHz}(\lambda \approx 1 \mathrm{dm})$ the signal amplitudes found for CMB distorted spectra well below FIRAS constraints are significantly larger than the estimates of the background from extragalactic sources fainter than some tens of nJy. At decreasing frequencies FF distortion amplitude increases but, at the same time, source confusion noise may represent a serious problem, possibly preventing the achievement of the faint detection threshold necessary to have a source contribution to the background significantly less than the CMB distortion amplitude.
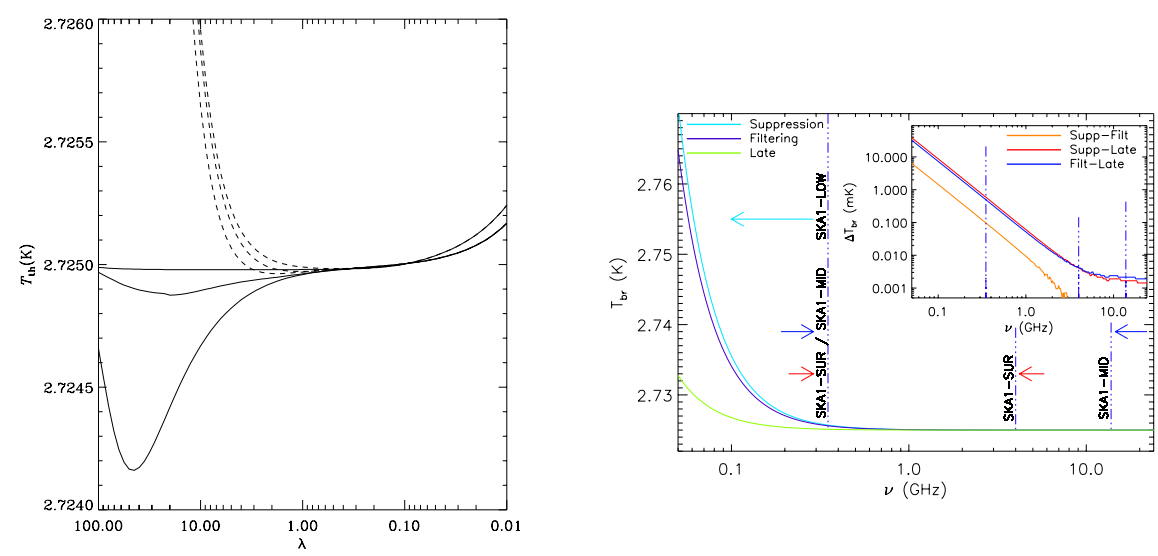

Fig. 9. Left panel: distorted spectra in equivalent thermodynamic temperature vs. $\lambda(\mathrm{cm})$ with late energy injection $\Delta \varepsilon / \varepsilon_{i}=5 \times 10^{-6}$ plus an early/intermediate energy injection $\Delta \varepsilon / \varepsilon_{i}=$ $5 \times 10^{-6}(\sim 20$ times smaller than current upper limits) at the "time" Comptonization parameter $y_{h}=5,1,0.01$ (bottom to top; the cases at $y_{h}=5$ and 1 are very similar at short $\lambda$; solid lines) plus a FF distortion with $y_{B}=10^{-6}$ (dashes). $y_{h}=y$ with $T_{e}=T_{C M B}$ when the integral is computed from the energy injection time to the current time. Right panel: FF distortion in SKA2 frequency range by two astrophysical reionization histories (a late phenomenological model is also displayed for comparison). Inset: models absolute differences; vertical lines: ranges of SKA1 configurations. From Ref. [141].

SKA will trace the neutral hydrogen distribution and the neutral-to-ionized transition state at the reionization epoch through the $21-\mathrm{cm}$ line. It could trace the development of ionized material directly by looking for $\mathrm{FF}$ emission from ionized halos. The expected signal can be derived by reionization models through both semi- 
analytical methods $\frac{144}{144}$ and numerical simulations $\frac{145}{10}$ Dedicated high resolution sky areas observations allow to distinguish $\mathrm{FF}$ distortion by ionized halos rather than by diffuse ionized IGM. SKA should be able to detect up to $\sim 10^{4}$ individual FF emission sources with $z>5$ in $1 \mathrm{deg}^{2}$ discerning ionized halos or diffuse ionized IGM FF distortions. Thus, the precise mapping of individual halos represents an interesting goal for the excellent imaging capabilities of SKA.

Acknowledgements - The authors that are members Planck Collaboration warmly thank the Planck Collaboration for numberless and constructive conversations on the subjects discussed here. Some of the results in this paper have been derived using the HEALPix ${ }^{123}$ package. We acknowledge the use of the NASA Legacy Archive for Microwave Background Data Analysis (LAMBDA) and of the ESA Planck Legacy Archive. CB, AG, ML and TT acknowledge partial support by ASI/INAF Agreement 2014-024-R.0 for the Planck LFI Activity of Phase E2.

\section{References}

1. Planck Collaboration, submitted to Astron. and Astrophys., arXiv:1502.01589 (2015).

2. S. Das, T. Louis, M.R. Nolta, et al., J. Cosm. and Astrop. Physics 04, article id. 014 (2014).

3. POLARBEAR Collaboration, The Astrophys. J. 794, 171 (2014).

4. E.M. George, C.L. Reichardt, K.A. Aird, et al., The Astrophys. J. 799, 177 (2015).

5. BICEP2/Keck and Planck Collaborations, Physical Review Letters 114, 101301 (2015).

6. R.K. Sachs and A.M. Wolfe, The Astrophys. J. 147, 73 (1976).

7. M.J. Rees and D.W. Sciama, Nature 217, 511 (1968).

8. E. Martínez-González, J.L. Sanz, J. Silk, The Astrophys. J. 355, L5 (1990).

9. R.G. Crittenden and N. Turok, Phys. Rev. D 76, 575 (1996).

10. W. Hu, Phys. Rev. D 65, 023003 (2002).

11. M. Kamionkowski, Phys. Rev. D 54, 4169 (1996).

12. S. Boughn and R. Crittenden, Nature 427, 45 (2004).

13. Planck Collaboration, submitted to Astron. and Astrophys., arXiv:1502.01595 (2015).

14. P. Vielva, E. Martínez-González, M. Tucci, Mon. Not. R. Astron. Soc. 365, 891 (2006).

15. H. Li and J. Xia, J. Cosm. and Astrop. Physics 04, article id. 026 (2010).

16. Planck Collaboration, Astron. and Astrophys. 571, A19 (2014).

17. Planck Collaboration, submitted to Astron. and Astrophys., arXiv:1502.05956 (2015).

18. Planck Collaboration, submitted to Astron. and Astrophys., arXiv:1502.01591 (2015).

19. R.B. Barreiro, P. Vielva, C. Hernandez-Monteagudo, E. Martínez-González, IEEE Journal of Selected Topics in Signal Processing 2, 747 (2008).

20. M. Frommert and T.A. Enßlin, Mon. Not. R. Astron. Soc. 395, 1837 (2009).

21. R. Laureijs, J. Amiaux, S. Arduini, et al., arXiv:1110.3193 (2011).

22. N. Benítez, R. Dupke, M. Moles, et al., arXiv:1403.5237|(2014).

23. LSST Dark Energy Science Collaboration, arXiv:1211.0310 (2012).

24. B.R. Granett, M.C. Neyrinck, I. Szapudi, The Astrophys. J. 683, L99 (2008).

25. G. Cabass, M. Gerbino, E. Giusarma, et al., Phys. Rev. D 92, 063534 (2015).

26. G. Hinshaw, et al., [WMAP Collaboration], The Astrophys. J. Suppl. 208, 19 (2013).

27. Planck Collaboration, Astron. and Astrophys. 571, A16 (2014). 
28. C.J. Copi, D. Huterer, D.J. Schwarz, G.D. Starkman, Advances in Astronomy 2010, 847541 (2010).

29. G. Hinshaw, A.J. Banday, C.L. Bennett, et al., The Astrophys. J. 464, L25 (1996).

30. D.N. Spergel, et al., [WMAP Collaboration], The Astrophys. J. Suppl. 148, 175 (2003).

31. A. Bernui, T. Villela, C.A. Wuensche, R. Leonardi, I. Ferreira, Astron. and Astrophys. 454, 409 (2006).

32. C. Copi, D. Huterer, D. Schwarz, G. Starkman, Phys. Rev. D 75, 023507 (2007).

33. C.J. Copi, D. Huterer, D.J. Schwarz, G.D. Starkman, Mon. Not. R. Astron. Soc. 399, 295 (2009).

34. G. Efstathiou, Y.Z. Ma, D. Hanson, Mon. Not. R. Astron. Soc. 407, 2530 (2010).

35. D. Sarkar, D. Huterer, C.J. Copi, G.D. Starkman, D.J. Schwarz, Astropart. Phys. 34, 591 (2011).

36. A. Gruppuso, Mon. Not. R. Astron. Soc. 437, 2076 (2014).

37. C.J. Copi, D. Huterer, D.J. Schwarz, G.D. Starkman, Mon. Not. R. Astron. Soc. 451, 2978 (2015).

38. A. Gruppuso, P. Natoli, F. Paci, et al., J. Cosm. and Astrop. Physics 07, article id. 047 (2013).

39. Planck Collaboration, Astron. and Astrophys., available on line, DOI: http://dx.doi.org/10.1051/0004-6361/201526681, arXiv:1502.05956 (2015).

40. A. Gruppuso and A. Sagnotti, IJMPD 24, 1544008 (2015).

41. A. Gruppuso, N. Kitazawa, N. Mandolesi, P. Natoli, A. Sagnotti, arXiv:1508.00411 (2015).

42. E. Dudas, N. Kitazawa, S. P. Patil, A. Sagnotti, J. Cosm. and Astrop. Physics 05, article id. 012 (2012).

43. N. Kitazawa and A. Sagnotti, J. Cosm. and Astrop. Physics 04, article id. 017 (2014); N. Kitazawa and A. Sagnotti, EPJ Web Conf. 95, 03031 (2015); N. Kitazawa and A. Sagnotti, Mod. Phys. Lett. A 30, 1550137 (2015).

44. E. Dudas, N. Kitazawa, A. Sagnotti, Phys. Lett. B 694, 80 (2010).

45. S. Sugimoto, Prog. Theor. Phys. 102, 685 (1999); I. Antoniadis, E. Dudas, A. Sagnotti, Phys. Lett. B 464, 38 (1999); C. Angelantonj, Nucl. Phys. B 566, 126 (2000); G. Aldazabal and A. M. Uranga, JHEP 9910, article id. 024 (1999); C. Angelantonj, I. Antoniadis, G. D'Appollonio, E. Dudas, A. Sagnotti, Nucl. Phys. B 572, 36 (2000).

46. Planck Collaboration, submitted to Astron. and Astrophys., arXiv:1502.02114 (2015).

47. U.H. Danielsson, Phys. Rev. D 66, 023511 (2002).

48. T. Biswas, A. Mazumdar, A. Shafieloo, Phys. Rev. D 82, 123517 (2010).

49. R. Flauger, L. McAllister, E. Pajer, A. Westphal, G. Xu, J. Cosm. and Astrop. Physics 06, article id. 009 (2010).

50. A. A. Starobinsky, JETP Lett. 55, 489 (1992); [Pisma Zh. Eksp. Teor. Fiz. 55, 477 (1992)].

51. G. Shiu and J. Xu, Phys. Rev. D 84, 103509 (2011).

52. J.A. Adams, B. Cresswell, R. Easther, Phys. Rev. D 64, 123514 (2001).

53. F. Beutler, et al., [BOSS Collaboration], Mon. Not. R. Astron. Soc. 443, 1065 (2014).

54. N. Bartolo, E. Komatsu, S. Matarrese, A. Riotto, Phys Rep. 402, 103 (2004).

55. X. Chen, Advances in Astronomy 2010, article id. 638979 (2010).

56. D. Babich, P. Creminelli, M. Zaldarriaga, J. Cosm. and Astrop. Physics 08, article id. 009 (2004).

57. V. Acquaviva, N. Bartolo, S. Matarrese, A. Riotto, Nuclear Physics B 667, 119 (2003).

58. J. Maldacena, Journal of High Energy Physics 05, article id. 013 (2003). 
59. X. Chen, M.-x. Huang, S. Kachru, G. Shiu, J. Cosm. and Astrop. Physics 01, article id. 002 (2007).

60. E. Silverstein and D. Tong, Phys. Rev. D 70, 103505, (2004).

61. C. Cheung, A.L. Fitzpatrick, J. Kaplan, L. Senatore, P. Creminelli, Journal of High Energy Physics 03, article id. 014 (2008).

62. E. Komatsu, D.N. Spergel, B.D. Wandelt, The Astrophys. J. 634, 14 (2005).

63. D. Munshi and A. Heavens, Mon. Not. R. Astron. Soc. 401, 2406 (2010).

64. J.R. Fergusson, M. Liguori, E.P.S. Shellard, Phys. Rev. D 82, 023502, (2010).

65. J.R. Fergusson, M. Liguori, E.P.S. Shellard, J. Cosm. and Astrop. Physics 12, article id. 32 (2012).

66. M. Bucher, B. van Tent, C.S. Carvalho, Mon. Not. R. Astron. Soc. 407, 2193 (2010).

67. Planck Collaboration, Astron. and Astrophys. 571, A24 (2014).

68. Planck Collaboration, submitted to Astron. and Astrophys., arXiv:1502.01592 (2015).

69. T. Giannantonio, C. Porciani, J. Carron, A. Amara, A. Pillepich, Mon. Not. R. Astron. Soc. 422, 2854 (2012).

70. J.B. Muoz, Y. Ali-Hamoud, M. Kamionkowski, Phys. Rev. D 92, 083508 (2015).

71. E. Pajer and M. Zaldarriaga, Physical Review Letters 109, 021302 (2012).

72. G. Drexlin, V. Hannen, S. Mertens, C. Weinheimer, Adv. High Energy Phys. 2013, 293986 (2013).

73. O. Cremonesi and M. Pavan, Adv. High Energy Phys. 2014, 951432 (2014).

74. J. Lesgourgues and S. Pastor, Phys. Rept. 429, 307 (2006).

75. G.L. Fogli, E. Lisi, A. Marrone, et al., Phys. Rev. D 70, 113003 (2004); S. Dell'Oro, S. Marcocci, M. Viel, F. Vissani, J. Cosm. and Astrop. Physics 12, article id. 023 (2015)

76. A. Lewis and S. Bridle, Phys. Rev. D 66, 103511 (2002).

77. D.V. Forero, M. Tortola, J.W.F. Valle, Phys. Rev. D 90, 093006 (2014).

78. M.C. Gonzalez-Garcia, M. Maltoni, J. Salvado, T. Schwetz, Journal of High Energy Physics 1212, 123 (2012).

79. A. Osipowicz, et al., [KATRIN Collaboration], Letter of intent, hep-ex/0109033 (2001).

80. B. Alpert, et al., [HOLMES Collaboration], Eur. Phys. J. C 75, 112 (2015).

81. M. Agostini, et al., [GERDA Collaboration], Physical Review Letters 111, 122503 (2013).

82. C. Cattadori, (private communication); C. Cattadori (on behalf of the GERDA Collaboration), talk given at the Neutrino Oscillation Workshop (unpublished) (2014).

83. A. Pocar (on behalf of the EXO-200 Collaboration), talk given at the Neutrino Oscillation Workshop (unpublished) (2014).

84. J.B. Albert, et al., [EXO-200 Collaboration], Nature 510, 229234 (2014).

85. A. Smolnikov and P. Grabmayr, Phys. Rev. C 81, 028502 (2010); J. Kotila and F. Iachello, Phys. Rev. C 85, 034316 (2012); J. Barea, J. Kotila, F. Iachello, Phys. Rev. C 87, 014315 (2013).

86. H. Minakata, H. Nunokawa, A.A. Quiroga, Progress of Theoretical and Experimental Physics 03, article id. 033B0324 (2015).

87. F. Fritsch and J. Butland, SIAM Journal on Scientific and Statistical Computing $\mathbf{5}$, 300 (1984).

88. S. Gariazzo, C. Giunti, M. Laveder, J. Cosm. and Astrop. Physics 04, article id. 023 (2015).

89. Planck Collaboration, submitted to Astron. and Astrophys., arXiv:1507.02704 (2015).

90. G. Efstathiou, Mon. Not. R. Astron. Soc. 440, 1138 (2014).

91. F. Beutler, C. Blake, M. Colless, et al., Mon. Not. R. Astron. Soc. 416, 3017 (2011). 
92. A.J. Ross, L. Samushia, C. Howlett, et al., Mon. Not. R. Astron. Soc. 449, 835 (2015).

93. L. Anderson, et al., [BOSS Collaboration], Mon. Not. R. Astron. Soc. 441, 24 (2014).

94. E. Di Valentino, S. Gariazzo, E. Giusarma, O. Mena, Phys. Rev. D 91, 123505 (2015).

95. E. Giusarma, E. Di Valentino, M. Lattanzi, A. Melchiorri, O. Mena, Phys. Rev. D 90, 043507 (2014).

96. E. Di Valentino, E. Giusarma, M. Lattanzi, A. Melchiorri, O. Mena, Phys. Rev. D 90, 043534 (2014).

97. F. Fritsch and R. Carlson, SIAM Journal on Numerical Analysis 17, 238 (1980).

98. Planck Collaboration, Astron. and Astrophys. 571, A1 (2014).

99. Planck Collaboration, Astron. and Astrophys. 571, A15 (2014).

100. C.L. Bennett, et al., [WMAP Collaboration], The Astrophys. J. Suppl. 208, 20 (2013).

101. C.L. Reichardt, L. Shaw, O. Zahn, et al., The Astrophys. J. 755, 70 (2012).

102. C. Blake, E.A. Kazin, F. Beutler, et al., Mon. Not. R. Astron. Soc. 418, 1707 (2011).

103. W.J. Percival, et al., [SDSS Collaboration], Mon. Not. R. Astron. Soc. 401, 2148 (2010).

104. N. Padmanabhan, X. Xu, D.J. Eisenstein, et al., Mon. Not. R. Astron. Soc. 427, 2132 (2012).

105. K.S. Dawson, et al., [BOSS Collaboration], The Astron. J. 145, 10 (2013).

106. C. Heymans, E. Grocutt, A. Heavens, et al., Mon. Not. R. Astron. Soc. 432, 2433 (2013).

107. Planck Collaboration, Astron. and Astrophys. 571, A20 (2014).

108. K.A. Olive, et al., [Particle Data Group], Chin. Phys. C 38, 090001 (2014).

109. G. Pignol, IJMPA 30, 1530048 (2015).

110. O. Pisanti, A. Cirillo, S. Esposito, et al., Comput. Phys. Commun. 178, 956 (2008).

111. L. Salvati, L. Pagano, R. Consiglio, A. Melchiorri, arXiv:1507.07243 (2015).

112. A. Mucciarelli, L. Lovisi, B. Lanzoni, F.R. Ferraro, The Astrophys. J. 786, 14 (2014).

113. M. Peimbert, V. Luridiana, A. Peimbert, The Astrophys. J. 666, 636 (2007).

114. Y.I. Izotov, T.X. Thuan, N.G. Guseva, Mon. Not. R. Astron. Soc. 445, 778 (2014).

115. IAU Commission 40, Polarization Definitions, Transactions of the IAU, Vol. XVB, p. 166 (1974).

116. W.-T. Ni, Rep. Prog. Phys 73, 056901 (2010).

117. L.I. Schiff, Am. J. Phys. 28, 340 (1960).

118. W.-T. Ni, Phys. Rev. Lett. 38, 301 (1977).

119. S. di Serego Alighieri, IJMPD 24, 1530016 (2015).

120. S.M. Carroll, G.B. Field, R. Jackiw, Phys. Rev. D 41, 1231 (1990).

121. J. Aumont, L. Conversi, C. Thum, et al., Astron. and Astrophys. 514, A70 (2010).

122. B.G. Keating, M. Shimon, A.P.S. Yadav, ApJL 762, L23 (2013).

123. K.M. Gorski, E. Hivon, A.J. Banday, et al., The Astrophys. J. 622, 759 (2005).

124. C.G.R. Wallis, A. Bonaldi, M.L. Brown, R.A. Battye, Mon. Not. R. Astron. Soc. 453, 2058 (2015).

125. V.A. Kostelecký and M. Mewes, Phys. Rev. Lett. 87, 251304 (2001).

126. V.A. Kostelecký and M. Mewes, Phys. Rev. D 66, 056005 (2002).

127. T. de Zeeuw, R. Tamai, J. Liske, The Messenger 158, 3 (2014).

128. G.H. Sanders, Jour. Astrophys and Astron. 34, 81 (2013).

129. R.A. Bernstein, P.J. McCarthy, K. Raybould, et al., Proc. SPIE, Vol. 9145, 91451C (2014).

130. Z. Ahmed, M. Amiri, S.J. Benton, et al., Proc. SPIE, Vol. 9153, 1 (2014).

131. A. Gruppuso, M. Gerbino, P. Natoli, et al., arXiv:1509.04157v1 (2015).

132. M. Massardi, S.G. Burke-Spolaor, T. Murphy, et al., Mon. Not. R. Astron. Soc. 436, 
2915 (2013).

133. L. Testi and J. Walsh, The Messenger 152, 2 (2013).

134. J.P. Kaufman, B.G. Keating, B.R. Johnson, Mon. Not. R. Astron. Soc. 455, 1981 (2016).

135. R. Salvaterra and C. Burigana, Mon. Not. R. Astron. Soc. 336, 592 (2002).

136. J.C. Mather, E.S. Cheng, R.E. Jr. Eplee, et al., The Astrophys. J. 354, L37 (1990).

137. R.A. Sunyaev and Ya.B. Zeldovich, Astrophys. and Space Science 7, 20 (1970).

138. J.G. Bartlett and A. Stebbins, The Astrophys. J. 371, 8 (1991).

139. A. Kogut, D.J. Fixsen, D.T. Chuss, et al., J. Cosm. and Astrop. Physics 07, article id. 025 (2011)

140. R.A. Sunyaev and R. Khatri, IJMPD 22, 1330014 (2013).

141. C. Burigana, P. Alexander, C. Baccigalupi, et al., in Advancing Astrophysics with the Square Kilometre Array, Proceedings of Science, PoS(AASKA14), 149 (2014) http://pos.sissa.it/archive/conferences/215/149/AASKA14_149.pdf.

142. T. Trombetti and C. Burigana, Mon. Not. R. Astron. Soc. 437, 2507 (2014).

143. J.J. Condon, W.D. Cotton, E.B. Fomalont, et al., The Astrophys. J. 758, article id. $23(2012)$

144. P. Naselsky and L.Y. Chiang, Mon. Not. R. Astron. Soc. 347, 921(2004).

145. P.P. Ponente, J.M. Diego, R.K. Sheth, et al., Mon. Not. R. Astron. Soc. 410, 2353 (2011). 